\title{
Questes
}

\section{Enfin la fin du Moyen Âge ?...}

\section{Benoît Grevin}

\section{(2) OpenEdition}

\section{Journals}

\section{Édition électronique}

URL : http://journals.openedition.org/questes/4291

DOI : 10.4000/questes.4291

ISSN : 2109-9472

\section{Éditeur}

Les Amis de Questes

\section{Édition imprimée}

Date de publication : 10 juin 2016

Pagination : 1-12

ISSN : 2102-7188

\section{Référence électronique}

Benoit Grevin, «Enfin la fin du Moyen Âge?... », Questes [En ligne], 33 | 2016, mis en ligne le 15 juin

2016, consulté le 23 septembre 2020. URL : http://journals.openedition.org/questes/4291 ; DOI :

https://doi.org/10.4000/questes.4291 


\title{
Enfin la fin du Moyen Âge ?...
}

\author{
Benoît GRÉVIN \\ Université Paris 1 Panthéon-Sorbonne, LAMOP
}

On penserait en avoir fini avec la fin du Moyen Âge. Il y a déjà longtemps, on le sait, que Jacques le Goff a procédé avec un bel appétit, caractéristique de l'«Ogre historien », à l'annexion programmatique d'à peu près toute l'histoire moderne (et d'une fraction notable de l'histoire antique et contemporaine...) au domaine d'une médiévistique rêvée comme une histoire totalisante, s'étendant jusqu'à Napoléon, voire jusqu'au monde de ceux de nos arrière-grands-parents qui transmettaient encore dans leurs terroirs des cultures traditionnelles. Cette opération, visant moins à faire la guerre aux historiens antiquisants et modernistes qu'à souligner la nécessité de penser l'histoire dans le temps long, n'était elle-même que la manifestation la plus apparente en France d'un désir général d'aérer les cadres chronologiques de l'histoire, désir qui a également conduit à la création de temporalités alternatives, chevauchant différentes périodes, telles que l'antiquité tardive ou, dans l'historiographie de langue anglaise, l'early modern history. Comprendre, avec les outils de l'historien, renforcés par ceux de l'anthropologue, le développement, les ruptures et les continuités de l'histoire européenne, comme d'ailleurs de l'histoire mondiale, nécessitait en effet de prendre ses distances avec un découpage traditionnel dont l'arbitraire était peut-être un peu moins prononcé pour la fin du Moyen Âge que pour la fin de l'antiquité (qui lit encore 476 comme la fin de l'empire romain? 1492 marque bien en revanche, tout de même, la découverte par les 
Européens de mondes nouveaux...), mais qui n'en restait pas moins pesamment européo-centré, et frisait l'anachronisme, dès l'âge de la décolonisation. L'âge du milieu? Par rapport à qui et à quoi ? Le Moyen Âge, en tout cas un Moyen Âge qui s'interrompt comme par magie à la fin $d u X V^{\mathrm{e}}$ siècle, ne veut pas dire grand-chose à l'aune de temporalités historiques non-européennes. Il a été traduit en arabe par l'expression «al qurūn al-wusțāa » القرون الوسطى, : lit. «les siècles moyens »), dans un décalque sémantique qui ne résout guère le problème de son utilisation pour l'histoire des mondes islamiques. Pourquoi, en effet, qualifier d'âge intermédiaire un temps qui, du point de vue de l'Islam, est essentiellement un âge des commencements, des «premières et des secondes grandeurs »? Les historiens japonais, eux, ont volontiers emprunté la notion, qui leur permettait de souligner le parallélisme nippoeuropéen d'un âge de la modernité étatique venant terminer un Moyen Âge (chûsei 中世) marqué ici et là par le féodalisme. Mais ce Moyen Âge japonais ne dure que quatre siècles, après une «antiquité » tardive et prestigieuse qui s'étend jusqu'en 1160, et il se clôt en décalage d'un bon siècle avec notre Moyen Âge européen, l'installation du shôgunat Tokugawa, cadre institutionnel des «modernités » japonaises, s'achevant à l'orée du XVII ${ }^{e}$ siècle. Il s'agissait ici de faire un usage efficient, non servile, d'une notion certes empruntée à l'Europe au plus fort du courant d'européanisation de Meiji, mais entendue, de manière intelligente, comme un concept opératoire devant être adapté aux scansions de l'histoire locale. Quant à la Chine, les caractéristiques de « modernité » économique, administrative et culturelle qui s'affirment dès l'époque des «Cinq royaumes et des dix dynasties» et surtout de la dynastie Song excluaient que les historiens du $\mathrm{Xx}^{\mathrm{e}}$ siècle, japonais ou chinois, acceptassent de se plier au découpage chronologique européen. L'instrument a été ici retourné par une partie de l'historiographie extrême- 
asiatique, suivie par nombre de chercheurs européens ou américains, l'utilisation du concept de Moyen Âge pour le seul premier millénaire par certains historiens (par exemple de la chute des Han postérieurs à celle des Tang : c. 200-c. 900, mais aussi dès la création de l'Empire, à la fin $\mathrm{du}$ troisième siècle avant notre ère...) venant souligner en creux la modernité de la Chine dès avant l'ère mongole'

En Europe même, en dépit d'un apparent consensus, la pesanteur des représentations et des histoires nationales imprime sa marque et change la donne de pays à pays, malgré l'évidence proclamée de la césure constituée par les «découvertes » de l'Amérique et par la Réforme. Pour les Italiens en particulier, les Tre- et Quattrocento, de 1300 à 1500, sont des siècles de transition qu'il est difficile de qualifier de médiévaux, et que l'on repousse déjà vers la modernité. Ces choix motivés par l'empreinte de la construction idéologique humaniste, persistante dans l'historiographie à travers les âges, recoupent ceux des early modern studies d'une partie de la recherche de langue anglaise, qui fondent en un seul bloc les derniers siècles du Moyen Âge et tout ou partie de l'époque moderne. Ce n'est d'ailleurs pas par hasard que les Renaissance studies, qui peinent à s'imposer en France comme une discipline propre, sont partie intégrantes de maint cursus universitaire outre-Atlantique. Les perceptions italiennes et anglophones se rejoignent ici. Au pays de Voltaire et de Michelet, le conditionnement par les représentations humanistes s'opère en revanche dans un sens contraire, avec une grande rigidité dans la scansion d'un «avant » et d'un «après »1492/1500/1515. $\mathrm{Au}$ niveau académique, avec tout ce que ce découpage induit d'automatismes et d'habitudes disciplinaires, mais aussi d'intériorisation inconsciente, le $\mathrm{XV}^{\mathrm{e}}$ siècle est médiéval, le $\mathrm{XVI}$ e siècle, moderne : tout juste

\footnotetext{
${ }^{1}$ Sur les problèmes de définition du Moyen Âge par les sinologues, voir Cho-Yun Hsu, «Chinese Periodisation», dans Encyclopedia of Social History, dir. Peter N. Stearns, New York/Londres, Garland, 1994, p. 161-163.
} 
les règnes de Charles VIII et Louis XII forment-ils un modeste sas de trente ans (1483-1515) qui peut être aménagé comme un espace intermédiaire.

Notre transition du Moyen Âge à l'époque moderne aurait pourtant pu se construire dans une respiration historiographique ample, allant de la fin de la guerre de Cent ans et de la chute de Constantinople jusqu'à la diffusion progressive du protestantisme dans le royaume. Ce laps de temps, formant une zone chronologique considérable, de 1450 à 1560 , pourrait être lu comme un siècle alternatif, domaine commun au Moyen Âge et à l'époque moderne. La vision tranchante d'une renaissance culturelle remontant, tel un éclair, à travers les premières descentes des guerres d'Italie, de Naples, Rome, Florence et Venise vers la Loire et Paris, s'est a contrario imposée très tôt, dès l'historiographie classique et romantique. Elle a renforcé, avec tout le poids d'une construction culturelle dépendante d'une certaine image de la Renaissance, l'idée d'une France basculant brutalement dans la modernité par une sorte de réveil culturel. À côté des temporalités impériales de 1453, océaniques de 1492, religieuses de 1517, il existe donc également une temporalité française qui choisit pour «sa » fin du Moyen Âge la date de 1494, liée à l'expédition napolitaine de Charles VIII. Michelet a poétisé et problématisé ce basculement, en le renforçant d'un balancement dialectique : après ce premier «lever de rideau » du monde moderne, le «bonhomme Louis XII », successeur de Charles VIII, fut dépeint par lui comme une sorte d'ultime représentant d'une culture des fabliaux et des soties médiévales, costumé en un Grandgousier anachronique qui ne pouvait que retarder l'avènement désormais irrésistible d'une modernité s'enflant à chaque retour d'Italie. Le début du règne du jeune François $\mathrm{I}^{\mathrm{er}}$ faisait, dans un troisième temps, définitivement basculer le pays dans une temporalité différente, symbolisée aussi bien par Jacques Cartier 
(un Colomb en réduction...) que par Rabelais ou par l'appel au Primatice et à Léonard de Vinci. Ces visions, superbes et caricaturales, sont proprement an-historiques, presque purement rhétoriques. Quoi de plus « moderne », en un certain sens que la tentative de construction d'un État franco-italien par Louis XII ? Quoi de plus médiéval que les disputes entre François I ${ }^{\mathrm{er}}$ et Charles Quint sur les suzerainetés des Flandres et de Bourgogne? Elles ont pourtant profondément marqué notre imaginaire collectif d'historiens, car «finir le Moyen Âge », c'était entrer dans une «modernité » qui, qu'on l'appelle renaissance ou non, continue d'imposer, parfois inconsciemment, une représentation de l'«avant» comme un ailleurs lointain, marqué par la distance anthropologique, par une relative fixité, face à un «après » plus ou moins explicitement affecté d'un dynamisme supérieur, d'une fluidité supposée l'ancêtre de nos contemporanéités et de leurs accélérations. La fin du Moyen Âge, déjà présentée comme un temps d'accélération relative dans la diffusion des savoirs, avec les multiplications des usages de l'écrit, l'emballement des copies manuscrites, aurait ainsi été le prologue à l'accélération plus violente de la communication symbolisée par l'invention (quelques siècles après la Chine, tout de même) et la diffusion de l'imprimerie. Il s'agit là d'un autre cliché, recouvrant celui-là un concept pleinement opératoire pour penser la fin du Moyen Âge, car l'invention, le perfectionnement et la diffusion en Europe de l'imprimerie fut un phénomène de longue durée, s'étalant sur trois générations (quand copie-t-on les derniers grands manuscrits enluminés ? Très tard, en plein $\mathrm{XVI}^{\mathrm{e}}$ siècle), qui convient bien à l'étude d'une zone de transition entre Moyen Âge et modernité.

Cette vision d'une accélération correspondant à une fin possible du Moyen Âge n'est sans doute pas totalement fausse : le monde se connecte, au moins d'un point de vue européen, de manière foudroyante, entre 1470 et 1530. Il existe d'ailleurs d'autres connections contemporaines, comme 
la simplification radicale opérée sur l'échiquier de l'Islam par la mondialisation de l'Empire ottoman entre 1450 et 1550, de Budapest à l'océan indien, de l'Algérie à l'Ukraine. Elle donne en quelque sorte au début de l'époque moderne une empreinte dynamique spécifique, porteuse de téléologie. Le succès d'une «histoire connectée » qu'affectionnent désormais «quinzième-» et «seiziémistes » est un symptôme de cette tentation de rétro-projeter nos obsessions actuelles d'un monde unifié par l'information en interrogeant selon cette grille le passé. Et, après la "parenthèse mongole », quoi de plus conforme à cette vision que la grande mise en réseau des années 1500 ? Mais penser le Moyen Âge comme statique par opposition à une modernité dynamique ne veut pas dire grand-chose du point de vue de l'histoire événementielle, culturelle, religieuse, si on veut bien envisager certains aspects de l'histoire européenne des $\mathrm{XIV}^{\mathrm{e}}$ et $\mathrm{XV}^{\mathrm{e}}$ siècles. Reconfigurations étatiques vertigineuses, aussi bien à l'Ouest (double monarchie franco-anglaise, puissance bourguignonne, ascension des États-cités italiens) qu'à l'Est du continent (constructions polono-lituaniennes, Luxembourg, Mitteleuropa de Matthias Corvin...), montagnes russes démographiques (la peste et ses suites...), implosion (le Grand Schisme) et recomposition d'une Église qui ne réussit plus à éradiquer des mouvements contestataires (hussitisme), diffusion rapide de nouveaux modèles culturels (l'humanisme, déjà...), tout cela fait de l'Europe de «l'automne du Moyen Âge ${ }^{2}$ » un temps à l'opposé du statisme dans lequel la combinaison d'une approche peut-être plus anthropologique et de la pression des clichés sur le «sombre Moyen Âge » persistant à travers le médiévalisme risquerait d'enfermer l'histoire du bas Moyen Âge. La différence qui sépare les derniers temps du Moyen Âge, jusque vers la fin

\footnotetext{
${ }^{2}$ Pour employer un concept inventé par Joseph Huizinga, L'Automne du Moyen Âge [1919], Paris, Payot, coll. « Petite bibliothèque », 2002.
} 
$\mathrm{du} \mathrm{XV}^{\mathrm{e}}$ siècle pour suivre le «modèle » français, et ceux de l'histoire moderne, serait peut-être plutôt que ces reconfigurations incessantes des $\mathrm{XIV}^{\mathrm{e}}$ et $\mathrm{XV}^{\mathrm{e}}$ siècles sont encore trop éloignées des cadres de l'Europe contemporaine pour servir de soubassement efficace à une vision téléologique du devenir européen. Une partie des constructions culturelles et étatiques du centre-est européen est ainsi balayée par l'avance ottomane au début du XVI ${ }^{\mathrm{e}}$ siècle : il ne reste rien, vers 1550, des «modernités » de l'«empire hongrois» de Matthias Corvin ${ }^{3}$. Toute la dynamique de l'interaction entre humanisme et pensée religieuse est profondément altérée par la Réforme et par ses suites : la modernité confessionnelle d'Érasme repousse les méditations chrétiennes d'un Pétrarque en plein Moyen Âge. Des recompositions étatico-dynastiques, comme la formation de la monarchie universelle Habsbourg, avec ses deux ailes hispano-italoflamande et mitteleuropéenne, ou l'explosion de l'union scandinave, viennent rebattre de fond en comble les cartes du politique. Il y a donc peut-être moins, au fond, accélération, qu'un ensemble de nouvelles orientations, radicalement imprévisibles en 1450, qui ont donné une direction alternative à l'histoire européenne, suggérant des combinaisons culturelles ou politiques très éloignées de celles qui semblaient se dessiner vers 1420, voire encore vers 1470. En amont, toutefois, de la systématisation des modes et procédures d'expérimentation qui marquera le début véritable de l'âge scientifique au XVII ${ }^{\mathrm{e}}$ siècle, doit-on vraiment qualifier ce nouveau monde de moderne? C'est loin d'être un choix évident, comme l'a montré Jérôme Baschet, en annexant le XVI siècle des structurations coloniales ibériques à l'histoire médiévale dans

\footnotetext{
${ }^{3}$ Sa construction politique préfigure territorialement l'Empire Habsbourg, mais avec une solution de continuité due à l'occupation turque. C'est par un hasard historique ou géopolitique que l'Autriche de Charles VI recouvre en grande partie la Hongrie expansionniste de Matthias Corvin, avec ses prolongements silésiens et basautrichiens, à moins de considérer, comme beaucoup l'ont fait, que la géographie impose ses logiques à l'histoire.
} 
La Civilisation féodale; de l'an mil à la colonisation de l'Amérique ${ }^{4}$ La proposition de refuser dans la pratique concrète de l'historien, non seulement du culturel, mais aussi du social, du politique et de l'institutionnel, la coupure de l'an 1500, ne conduit toutefois pas tant à effacer le couple conceptuel «médiéval/moderne», qu'à repousser l'entrée dans la modernité jusqu'au moment où le chercheur décide d'opérer le basculement entre les deux temporalités. Le médiéviste européen (de culture euro-américaine...) rejoint ici, à la faveur d'une étude décentrée examinant la mise en place de mécanismes institutionnels inspirés de la féodalité pour gouverner les Amériques, les nipponologues qui ont accepté de «jouer le jeu» du Moyen Âge, mais en refusant les cadres traditionnels assignés à la notion. Les potentialités d'un tel exercice se révèlent telles, en histoire culturelle, politique ou économique, qu'on ne peut qu'appeler de ses vœux sa multiplication. Il s'agirait alors, que l'on «médiévalise » le début de l'époque moderne, ou que l'on modernise la fin du Moyen Âge, de ne «jamais finir» le Moyen Âge, la barrière chronologique étant sans cesse déplacée au gré du questionnement d'historiens qui définiraient eux-mêmes, en fonction de leurs critères de recherche, l'entrée dans la modernité. De cette manière, l'étude du Moyen Âge engloberait bien, potentiellement, celle de quasiment toute la modernité des $\mathrm{XVI}^{\mathrm{e}}, \mathrm{XVII}^{\mathrm{e}}$ et $\mathrm{XVIII}{ }^{\mathrm{e}}$ siècles. Le Moyen Âge, s'étendant potentiellement à différents aspects ou enquêtes de l'époque moderne, ne finirait définitivement comme sujet que quand les propres acteurs de l'histoire l'objectiveraient, avec l'apparition progressive, entre la génération de Voltaire et celle des premiers Romantiques, de la réflexion historique sur le Moyen Âge, et du médiévalisme. Le Moyen Âge ne mourrait ainsi à lui-même, en quelque sorte, qu'en s'inventant, les

\footnotetext{
4 Jérôme Baschet, La Civilisation féodale; de l'an mil à la colonisation de l'Amérique, Paris, Aubier, coll. «Collection historique », 2004.
} 
spécialistes du médiévalisme (souvent eux-mêmes des médiévistes de formation) prenant le relais pour l'Europe de l'époque contemporaine, riche d'un rapport au Moyen Âge qui ne s'exerce plus sur le mode de la continuation, mais de la nostalgie et de la distanciation ${ }^{5}$...

«Finir le Moyen Âge » serait donc en finir avec la fin traditionnelle du Moyen Âge, en acceptant le jeu d'une histoire du temps long et de ses transitions (1300-1700), ou même d'un temps court alternatif (14501550), qui refuse les incommodes arbitrages disciplinaires, ou qui en propose d'autres. Pourquoi, en effet, ne pas mettre l'accent sur d'autres coupures, a contrario de nos pesanteurs disciplinaires ? Quel médiéviste, par exemple, n'a pas ressenti la différence méthodologique abyssale entre la pratique, les questions, parfois les méthodes des spécialistes du plus haut Moyen Âge (temps mérovingiens pour la France), désormais bien assis dans une étude des continuités avec le monde romain caractéristiques de l'antiquité tardive, et la recherche concernant les XIII $\mathrm{XIV}^{\mathrm{e}}$ ou $\mathrm{XV}^{\mathrm{e}}$ siècles? Que l'historien des trois derniers siècles du Moyen Âge ait souvent plus à faire avec ses collègues modernistes, spécialistes des $\mathrm{XVI}^{\mathrm{e}}$ et $\mathrm{XVII}{ }^{\mathrm{e}}$ siècles, qu'avec les médiévistes des Dark Ages anglais ou du monde mérovingien est un truisme, truisme qu'il faut pourtant rappeler : dans nos universités et nos centres de recherche, c'est encore et toujours le regroupement «pan-médiéval » qui est privilégié, le dialogue autour de la barrière de 1500 étant éventuellement encouragé comme un effort interdisciplinaire, malgré sa plus grande évidence.

En dépit de notre volonté d'en finir avec la «fin du Moyen Âge», et de la possibilité très concrète de le faire, il reste donc peut-être raisonnable de tenir compte de cette pesanteur institutionnelle, qui n'est

\footnotetext{
${ }^{5}$ Sur le médiévalisme, voir à présent Tommaso di Carpegna Falconieri, Médiéval et militant. Penser le contemporain à travers le Moyen Âge, Paris, Publications de la Sorbonne, coll. « Histoire ancienne et médiévale », 2015.
} 
pas, a priori, appelée à disparaitre en France dans les prochaines années, mais, au mieux, à s'atténuer. Et l'on doit donc sans doute se résigner provisoirement à feindre de trouver une valeur heuristique particulière à la « coupure », ou plutôt à l'inflexion des années 1450-1530, qui se justifierait malgré tout, sous le triple poids de la formation des empires mondiaux ibériques, de la scission confessionnelle et des mutations étatiques. Si l'on accepte de jouer ce jeu, en dépit de toutes les limites, de toutes les possibilités alternatives déjà soulignées, ce serait encore une fois à condition de ne reconnaître à cette césure qu'une valeur relative, devant être sans cesse remise en question face à d'autres césures historiques potentiellement tout aussi importantes, et qui l'encadrent. Évoquons pour ne parler que de l'histoire de l'Europe (et de l'Islam le plus lié à l'Europe), en amont, la fin du beau Moyen Âge en 1300-1350, à la fois géopolitique (avancée ottomane, fins des États latins de Syrie), démographique, culturelle et étatique, ou en aval, le milieu du XVII ${ }^{\mathrm{e}}$ siècle (début du régime de généralisation et de diffusion accélérée des procédures scientifiques d'expérimentation à travers l'Europe, avec son cortège de révolutions techniques, apaisement relatif des conflits confessionnels, prodromes de la «seconde colonisation »). La césure de 1500, dotée d'une force d'inertie institutionnelle, plus en vertu d'une tradition bien ancrée, que d'un besoin réel, serait ainsi maintenue, mais comme l'un des seuils possibles d'une série de mutations entre lesquelles les historiens navigueraient, comme entre une série d'écluses. Il s'agirait en quelque sorte de la pause médiane, d'une scansion plus forte, dans un ensemble de transitions multipliables à l'infini. Il faudrait alors imaginer la «fin du Moyen Âge» comme une longue coda musicale, se développant sur quatre ou cinq siècles (1300 - ou déjà 1200 ? - 1650, voire 1700 ?), dans un mouvement où la phase des années 1470-1530 marquerait un point fort, un faisceau de mutations concentrant la 
réflexion, sans pour autant l'achever. Le poids de ces concepts étranges, « modernité » et « Moyen Âge », serait ainsi relativisé, car ils ne seraient plus que deux modes de lecture d'un patrimoine global, deux modes de présentation superposables d'une même histoire, perdant ainsi leur résonnance «statique » ou «dynamique», «archaïque » ou «progressiste ». Car la «modernité » du Moyen Âge, l' «archaïsme » de l'époque moderne, sont désormais des clichés si bien ancrés dans nos pratiques historiennes que nous devrions pouvoir en jouer pour éviter toute tentation de nous laisser influencer par la polarisation artificielle entre un avant lointain et un passé proche, conjurée par les deux notions, et qui persiste dans les représentations collectives. Le temps passe, après tout, pour nous aussi, et l'histoire moderne ne peut plus avoir la même signification, pour le chercheur ou l'honnête homme du XXI siècle, que celle qu'elle avait pour nos aïeux de la Belle Époque. Elle était pour eux «proto-histoire » de leurs modernités, souvenir encore trop proche pour supporter facilement l'épreuve de l'objectivation. Elle est elle-même devenue un «âge intermédiaire » dont le statut n'est plus celui d'une entrée dans la modernité, mais bien d'un passé antérieur. Elle s'est faite, à sa manière, Moyen Âge...

La série d'études recueillies et introduites dans ce volume de Questes par Pauline Guéna et Annabelle Marin prend à bras le corps la question de la fin du Moyen Âge en usant des deux grilles, large et étroite, traditionnelle et novatrice, ici suggérées. Certes, plusieurs des études d'histoire sociale, politique, culturelle réunies ici affrontent une temporalité qui est celle de la transition française habituelle de la fin du Moyen Âge vers l'époque moderne, avec une place privilégiée donnée aux dernières décennies $d u X V^{e}$ et aux premières années du XVI siècle. Toutefois, comme l'introduction le prouve éloquemment, le dispositif choisi l'est sans illusion sur la valeur heuristique de la coupure 
traditionnelle : il s'agit au contraire de réexaminer ce fameux basculement de la fin du $X v^{e}$ siècle, en revenant sur cette césure avec toute la distance que les avancées historiographiques, qu'une nouvelle philosophie de l'histoire, permettent d'apporter. Et certaines des enquêtes, plongeant au contraire dans le temps long du culturel, du politique, voire de l'archivistique, apportent le contre-point nécessaire pour penser les deux temporalités - courte et longue - de la fin du Moyen Âge. Jeu de déconstruction et de reconstruction à la fois d'un âge intermédiaire, mais aussi d'une transition idéalisée qu'il s'agit d'examiner de manière critique, comme une construction historiographique plutôt que comme une véritable entité, le dossier «finir le Moyen Âge» propose une lecture alternative de ces transitions, soulignant comment les deux adjectifs «moderne» et «médiéval» conditionnent encore nos lectures de l'histoire de ces années, suggérant comment il faut lutter contre les pesanteurs institutionnelles et les fausses évidences pour interroger ces chevauchements. Ce faisant, il suggère aussi comment retourner un cliché pour en faire un nouvel instrument heuristique, un instrument plus adapté pour étudier cette fin du Moyen Âge qui n'en finit jamais. 